\title{
IMPACT OF AGE IN HEALTH-RELATED QUALITY OF LIFE IN OLDER ADULTS WITH CANCER
}

\section{Impacto da idade na qualidade de vida relacionada à saúde em idosos com câncer}

\author{
Coeli Regina Carneiro Ximenes ${ }^{\mathrm{a}}$ (1) Anke Bergmann ${ }^{\mathrm{b}}$ (1), Jurema Telles de Oliveira Lima ${ }^{\mathrm{a}}$ (1), \\ Arlene Santos Cavalcantia ${ }^{\circledR}$, Murilo Carlos Amorim de Britto ${ }^{\circledR}$, \\ Maria Julia Gonçalves Melloa $\mathbb{B}$, Luiz Claudio Santos Thuler ${ }^{\mathrm{b}}$
}

\begin{abstract}
OBJECTIVE: To evaluate the impact of age in health-related quality of life (HRQoL) in older adults with cancer. METHODS: This was a cross-sectional study of 608 older adults diagnosed with cancer. Age groups were considered an independent variable. For the analysis of HRQoL, the mean scores of age groups were compared by analysis of variance and the Scheffé comparison test. For measuring the association between age and HRQoL, we used simple and multiple linear regression analyses. RESULTS: Cognitive function showed the highest scores (average $87.94 \pm 26.87$ ), while physical function showed the lowest ones (68.04 $\pm 28.63)$. The highest symptom score was observed for financial difficulties (34.21 \pm 39.06$)$, followed by pain (29.47 \pm 33.92$)$ and insomnia (28.51 \pm 37.03 ). After adjustment, we observed a decrease in physical function ( $p=0.028)$ and an improvement in emotional function ( $p=0.003$ ) with increasing age. CONCLUSIONS: In older patients with cancer, age negatively impacted physical function and positively impacted emotional function.
\end{abstract}

KEYWORDS: age distribution; older adults; neoplasms; quality of life.

OBJETIVO: Avaliar o impacto da idade na qualidade de vida relacionada à saúde (QVRS) em idosos com câncer. MÉTODOS: Estudo transversal envolvendo 608 idosos diagnosticados com câncer. Os grupos etários foram considerados uma variável independente. Para a análise da QVRS, os escores médios dos grupos etários foram comparados utilizando a análise de variância e o teste de Scheffé. Para medir a associação entre idade e QVRS, foram utilizadas análises de regressão linear simples e múltipla. RESULTADOS: A função cognitiva apresentou os maiores escores (média 87,94 $\pm 26,87$ ), enquanto a função física apresentou os menores (68,04 $\pm 28,63)$. O maior escore de sintomas foi observado em dificuldades financeiras $(34,21 \pm 39,06)$, seguido por dor $(29,47 \pm 33,92)$ e insônia $(28,51 \pm 37,03)$. Após o ajuste, observamos diminuição da função física $(p=0,028)$ e melhora da função emocional ( $p=0,003$ ) com o aumento da idade. CONCLUSÕES: Em pacientes idosos com câncer, a idade impactou negativamente a função física e positivamente a função emocional.

PALAVRAS-CHAVE: distribuição por idade; idoso; neoplasias; qualidade de vida.

alnstituto de Medicina Integral Professor Fernando Figueira - Recife (PE), Brazil.

'Instituto Nacional de Câncer José Alencar Gomes da Silva - Rio de Janeiro (RJ), Brazil.

Correspondence data

Anke Bergmann - Instituto Nacional de Câncer José Alencar Gomes da Silva - Rua André Cavalcanti, 37, 30 andar - Fátima - CEP: 20231050 - Rio de Janeiro (RJ), Brazil. E-mail: abergmann@inca.gov.br @Ankebergmann

Received on: 08/24/2020. Accepted on: 11/28/2020

How to cite this article: Ximenes CRC, Bergmann A, Lima JTO, Cavalcanti AS, Brito MC, Mello MJG, et al. Impact of age in health-related quality of life in older adults with cancer. Geriatr Gerontol Aging. 2021;15:e0210005. https://doi.org/10.5327/Z2447-212320212000106

https://doi.org/10.5327/22447-212320212000106 


\section{INTRODUCTION}

Population aging is a significant contributor to epidemiological transition and current morbidity and mortality profiles worldwide. ${ }^{1}$ The incidence of cancer is strongly related to aging. If current trends continue, by $2020,70 \%$ of all cancers will occur in people aged 65 years or older, further increasing the morbidity related to cancer in older adults., ${ }^{2,3}$

Although longevity is often correlated with increased morbidity, poor health is not necessarily a consequence of survival to older ages. Most studies that compare the health of older populations categorize chronological age into groups. The World Health Organization (WHO) ${ }^{2}$ considers the young old as those aged 60-74 years, the old old as those aged 75-84 years, and the oldest old as people aged 85 years or more. In Brazil, the Institute of Applied Economic Research (IPEA) ${ }^{4}$ considers that younger older adults are people between 60 and 70 years old, moderately older adults are those between 70 and 80 years old, and very older adults are aged 80 years or older.

In recent years, the assessment of health-related quality of life (HRQoL) in older adults with cancer has been recognized as an important component during all phases of the disease, from diagnosis to palliative care. ${ }^{5,6} \mathrm{HRQ}$ L can be defined as the individual's perception of their position in life in the context of the culture and value systems in which they live and their goals, expectations, standards, and concerns. ${ }^{7}$

The measurement of HRQoL should thus include components such as functional, cognitive, emotional, social, and economic status. ${ }^{7}$ Moreover, in patients with cancer, the problems and symptoms caused by the disease or its treatment should be evaluated. ${ }^{8}$ This assessment allows the recognition of vulnerable groups for individualized multidisciplinary care and the assessment of the impact, tolerability, and adherence to treatment regimens by the patients. ${ }^{8-10} \mathrm{HRQoL}$ has been used as a primary outcome in cancer trials, has been considered ancillary when implementing treatment options, and is a predictor of treatment complications and overall survival., ${ }^{611-14}$

The effects of age on HRQoL were studied in a systematic review with 6024 patients with cancer. ${ }^{13}$ The authors observed that the HRQoL domains may be influenced by age. In older patients, physical functioning and constipation were associated with worse perceptions, while social functioning, insomnia, and financial problems were associated with better ones. Optimal care for older patients with cancer should consider aspects of HRQoL. ${ }^{13}$

According to the $\mathrm{WHO},{ }^{2}$ by 2025 , Brazil will be the sixth country in the world in the number of older adults. This increase in average life expectancy, combined with other factors, has made cancer an important public health problem in the country. However, little is known in Brazil about at which age group the impacts produced by this disease and its treatments become the most important. In this context, this study aims to assess the demographic and clinical characteristics, as well as the HRQoL, of older people diagnosed with cancer according to age group.

\section{METHODS}

This cross-sectional study was performed at cancer diagnosis with patients who had a malignant neoplasm, were aged $\geq 60$ years, and were enrolled for oncologic treatment at Instituto de Medicina Integral Prof. Fernando Figueira, in Recife, Brazil from January 2015 to July 2016. Patients whose Mini Mental State Examination scores were lower than 23 were excluded, as well as patients who had already started systemic cancer treatment or radiotherapy.

Data were collected through interviews at the time of enrollment in the institution. Sociodemographic (ie, current age, sex, marital status, education, household income, self-reported skin color, alcohol consumption, and smoking habits) and clinical characteristics (ie, topography of cancer according to the 10th edition of the International Classification of Diseases [ICD 10], metastatic disease at diagnosis, Karnofsky Performance Scale [KPS], short form of the mini nutritional assessment [MNA-SF], and Charlson Comorbidity Index [CCI]) were collected. The age group at cancer diagnosis was considered an independent variable and was categorized according to the IPEA ${ }^{4}$ into: $60-64,65-69,70-74,75-79$, and $\geq 80$ years old.

For the evaluation of HRQoL (dependent variable), we used the European Organisation for Research and Treatment of Cancer Quality of Life Questionnaire (EORTC QLQ-C30), covering 5 functions (physical, global, cognitive, emotional, and social), global health, 3 symptom scales (fatigue, pain, and nausea/vomiting), and 6 additional symptom items (dyspnea, insomnia, loss of appetite, constipation, diarrhea, and financial difficulties). ${ }^{15}$ Questionnaire scores ranged from 0 to 100 and were calculated according to the protocol proposed by the EORTC QLQ-C $30 .{ }^{16}$ Regarding the functional scales and global health status, higher scores are related to better quality of life in the individual's perception; conversely, for symptom scales, higher scores correspond to a greater impact of that symptom, or worse quality of life. The questionnaire was translated into Portuguese and was validated for the Brazilian population. ${ }^{17}$

Our descriptive analysis was used means and standard deviations (SDs) for quantitative variables and absolute and relative frequencies for qualitative variables. Demographic and clinical characteristics, according to age group, were compared using Pearson's $\chi^{2}$ test; differences between categories were assessed using the $Z$-test. HRQoL was analyzed as having a normal distribution, as proposed by the EORTC QLQ-C30. ${ }^{16}$ 
For evaluating quality of life according to age group, differences between each mean score were calculated by analysis of variance (ANOVA) and the Scheffé comparison test. For all comparisons, $\mathrm{p}<0.05$ was considered statistically significant.

We used simple linear regression to evaluate the association between age and HRQoL in the older adult. We selected demographic and clinical variables with $p<0.20$ in the univariate analysis to identify possible adjustment variables and then conducted a multiple linear regression model through the Stepwise Forward method.In the final model, variables with $\mathrm{p}<0.05$ were retained. Data analysis was performed using SPSS, version 23.0.
All participants signed an informed consent form. This study was submitted to the National Cancer Institute (INCA) Research Ethics Committee under number 4412-14.

\section{RESULTS}

The study included 608 older adults with a mean age of 71.86 years $( \pm 7.44) ; 65-69$ years was the most common age interval (24\%). The demographic characteristics according to age group are shown in Table 1. A lower frequency of men in the group aged $<65$ years was observed when compared

Table 1. Overall characteristics of older adults diagnosed with cancer, according to age group $(N=608)$.

\begin{tabular}{|c|c|c|c|c|c|c|c|c|}
\hline \multirow[b]{2}{*}{ Characteristics } & \multirow[b]{2}{*}{ n (\%) } & \multicolumn{5}{|c|}{ Age group (years) n (\%) } & \multirow{2}{*}{$\begin{array}{l}\text { Differences } \\
\text { between } \\
\text { categories* }\end{array}$} & \multirow[b]{2}{*}{ p-value** } \\
\hline & & $\begin{array}{l}<65 \\
\text { (A) }\end{array}$ & $\begin{array}{l}65-69 \\
\text { (B) }\end{array}$ & $\begin{array}{l}70-74 \\
\text { (C) }\end{array}$ & $\begin{array}{l}75-79 \\
\text { (D) }\end{array}$ & $\begin{array}{l}\geq 80 \\
\text { (E) }\end{array}$ & & \\
\hline \multicolumn{9}{|l|}{ Sex } \\
\hline Male & $\begin{array}{c}309 \\
(50.82)\end{array}$ & $\begin{array}{c}42 \\
(37.16)\end{array}$ & $\begin{array}{c}72 \\
(49.32)\end{array}$ & $\begin{array}{c}82 \\
(56.95)\end{array}$ & $\begin{array}{c}56 \\
(51.34)\end{array}$ & $\begin{array}{c}57 \\
(58.16)\end{array}$ & $\mathrm{A}<\mathrm{B}=\mathrm{C}=\mathrm{D}=\mathrm{E}$ & \multirow{2}{*}{0.012} \\
\hline Female & $\begin{array}{c}299 \\
(49.18)\end{array}$ & $\begin{array}{c}71 \\
(62.84)\end{array}$ & $\begin{array}{c}74 \\
(50.68)\end{array}$ & $\begin{array}{c}62 \\
(43.05)\end{array}$ & $\begin{array}{c}51 \\
(47.66)\end{array}$ & $\begin{array}{c}41 \\
(41.84)\end{array}$ & $A>B=C=D=E$ & \\
\hline \multicolumn{9}{|l|}{ Lives with a partner } \\
\hline No & $\begin{array}{c}270 \\
(44.41)\end{array}$ & $\begin{array}{c}46 \\
(40.71)\end{array}$ & $\begin{array}{c}58 \\
(39.73)\end{array}$ & $\begin{array}{c}56 \\
(38.89)\end{array}$ & $\begin{array}{c}57 \\
(53.27)\end{array}$ & $\begin{array}{c}53 \\
(54.08) \\
\end{array}$ & $\mathrm{A}=\mathrm{B}=\mathrm{C}<\mathrm{D}=\mathrm{E}$ & \multirow{2}{*}{0.029} \\
\hline Yes & $\begin{array}{c}338 \\
(55.59)\end{array}$ & $\begin{array}{c}67 \\
(59.29)\end{array}$ & $\begin{array}{c}88 \\
(60.27)\end{array}$ & $\begin{array}{c}88 \\
(61.11)\end{array}$ & $\begin{array}{c}50 \\
(46.73)\end{array}$ & $\begin{array}{c}45 \\
(45.92)\end{array}$ & $A=B=C>D=E$ & \\
\hline \multicolumn{9}{|l|}{ Education } \\
\hline$\geq 4$ years of schooling & $\begin{array}{c}286 \\
(47.04)\end{array}$ & $\begin{array}{c}37 \\
(32.74)\end{array}$ & $\begin{array}{c}55 \\
(37.67)\end{array}$ & $\begin{array}{c}72 \\
(50.00)\end{array}$ & $\begin{array}{c}61 \\
(57.01)\end{array}$ & $\begin{array}{c}61 \\
(62.24)\end{array}$ & $A=B<C=D=E$ & \multirow{2}{*}{$<0.001$} \\
\hline$<4$ years of schooling & $\begin{array}{c}322 \\
(53.96)\end{array}$ & $\begin{array}{c}76 \\
(67.26)\end{array}$ & $\begin{array}{c}91 \\
(62.33)\end{array}$ & $\begin{array}{c}72 \\
(50.00)\end{array}$ & $\begin{array}{c}46 \\
(42.99)\end{array}$ & $\begin{array}{c}37 \\
(37.76)\end{array}$ & $A=B>C=D=E$ & \\
\hline \multicolumn{9}{|l|}{ Family income ${ }^{* * * *}$} \\
\hline$\leq 1$ minimum wage & $\begin{array}{c}152 \\
(25.00)\end{array}$ & $\begin{array}{c}27 \\
(23.89) \\
\end{array}$ & $\begin{array}{c}42 \\
(28.77) \\
\end{array}$ & $\begin{array}{c}36 \\
(25.00) \\
\end{array}$ & $\begin{array}{c}22 \\
(20.56) \\
\end{array}$ & $\begin{array}{c}25 \\
(25.51) \\
\end{array}$ & $A=B=C=D=E$ & \multirow{3}{*}{0.778} \\
\hline $1-2$ times the minimum wage & $\begin{array}{c}228 \\
(37.50)\end{array}$ & $\begin{array}{c}43 \\
(38.05) \\
\end{array}$ & $\begin{array}{c}58 \\
(39.73)\end{array}$ & $\begin{array}{c}52 \\
(36.11)\end{array}$ & $\begin{array}{c}38 \\
(35.51)\end{array}$ & $\begin{array}{c}37 \\
(37.76)\end{array}$ & $A=B=C=D=E$ & \\
\hline$\geq 2$ times the minimum wage & $\begin{array}{c}228 \\
(37.50)\end{array}$ & $\begin{array}{c}43 \\
(38.05)\end{array}$ & $\begin{array}{c}46 \\
(31.50)\end{array}$ & $\begin{array}{c}56 \\
(38.89)\end{array}$ & $\begin{array}{c}47 \\
(43.93)\end{array}$ & $\begin{array}{c}36 \\
(36.73)\end{array}$ & $A=B=E=C<D$ & \\
\hline \multicolumn{9}{|l|}{ Self-reported skin color } \\
\hline White & $\begin{array}{c}165 \\
(27.14)\end{array}$ & $\begin{array}{c}31 \\
(27.43)\end{array}$ & $\begin{array}{c}38 \\
(26.03)\end{array}$ & $\begin{array}{c}34 \\
(23.61)\end{array}$ & $\begin{array}{c}28 \\
(26.17)\end{array}$ & $\begin{array}{c}34 \\
(34.69)\end{array}$ & $A=B=C=D=E$ & \multirow{2}{*}{0.422} \\
\hline Not white & $\begin{array}{c}443 \\
(72.86) \\
\end{array}$ & $\begin{array}{c}82 \\
(72.57) \\
\end{array}$ & $\begin{array}{c}108 \\
(73.97)\end{array}$ & $\begin{array}{c}110 \\
(76.39)\end{array}$ & $\begin{array}{c}79 \\
(73.83) \\
\end{array}$ & $\begin{array}{c}64 \\
(65.31) \\
\end{array}$ & $A=B=C=D=E$ & \\
\hline \multicolumn{9}{|l|}{ Smoking } \\
\hline Current or former smoker & $\begin{array}{c}345 \\
(56.74)\end{array}$ & $\begin{array}{c}64 \\
(56.64) \\
\end{array}$ & $\begin{array}{c}71 \\
(48.63)\end{array}$ & $\begin{array}{c}85 \\
(59.03) \\
\end{array}$ & $\begin{array}{c}67 \\
(62.62)\end{array}$ & $\begin{array}{c}58 \\
(59.18)\end{array}$ & $B<D ; A=C=E$ & \multirow{2}{*}{0.202} \\
\hline Never smoked & $\begin{array}{c}263 \\
(43.26)\end{array}$ & $\begin{array}{c}49 \\
(43.36)\end{array}$ & $\begin{array}{c}75 \\
(51.37)\end{array}$ & $\begin{array}{c}59 \\
(40.97)\end{array}$ & $\begin{array}{c}40 \\
(37.38)\end{array}$ & $\begin{array}{c}10 \\
(40.82)\end{array}$ & $B>D ; A=C=E$ & \\
\hline \multicolumn{9}{|l|}{ Alcoholism } \\
\hline Current or former alcoholic & $\begin{array}{c}324 \\
(53.29)\end{array}$ & $\begin{array}{c}64 \\
(56.64)\end{array}$ & $\begin{array}{c}78 \\
(53.42)\end{array}$ & $\begin{array}{c}79 \\
(54.86)\end{array}$ & $\begin{array}{c}58 \\
(54.21)\end{array}$ & $\begin{array}{c}45 \\
(45.91)\end{array}$ & $A=B=C=D=E$ & \multirow{2}{*}{0.587} \\
\hline Never & $\begin{array}{c}284 \\
(46.71)\end{array}$ & $\begin{array}{c}49 \\
(43.36)\end{array}$ & $\begin{array}{c}68 \\
(46.56)\end{array}$ & $\begin{array}{c}65 \\
(45.14)\end{array}$ & $\begin{array}{c}49 \\
(45.79)\end{array}$ & $\begin{array}{c}53 \\
(54.08)\end{array}$ & $A=B=C=D=E$ & \\
\hline Total $^{* * *}$ & $\begin{array}{c}608 \\
(100.00)\end{array}$ & $\begin{array}{c}113 \\
(18.59)\end{array}$ & $\begin{array}{c}146 \\
(24.01)\end{array}$ & $\begin{array}{c}144 \\
(23.68)\end{array}$ & $\begin{array}{c}107 \\
(17.60)\end{array}$ & $\begin{array}{c}98 \\
(16.12)\end{array}$ & --- & --- \\
\hline
\end{tabular}

*Z-test; **Pearson's $\chi^{2}$ test; ${ }^{* * *}$ row percent; ${ }^{* * *}$ in 2015/2016, the minimum wage was U\$ 250/270 per month; statistically significant values are highlighted in bold. 
to women in the same age group (37.16 and $62.84 \%$, respectively; $\mathrm{p}=0.012$ ). Participants in the younger age groups lived with a partner with greater frequency than those in the older ones $(p=0.029)$. Furthermore, older adults aged up to 69 years had higher education levels when compared to the other groups ( $\mathrm{p}<0.001)$. There were no differences between age groups regarding family income, self-declared skin color, and tobacco and alcohol consumption.

Considering clinical characteristics (Table 2), older adults aged 80 or older had worse functional performance in the

Table 2. Clinical characteristics of older adults diagnosed with cancer, according to age group $(\mathrm{N}=608)$.

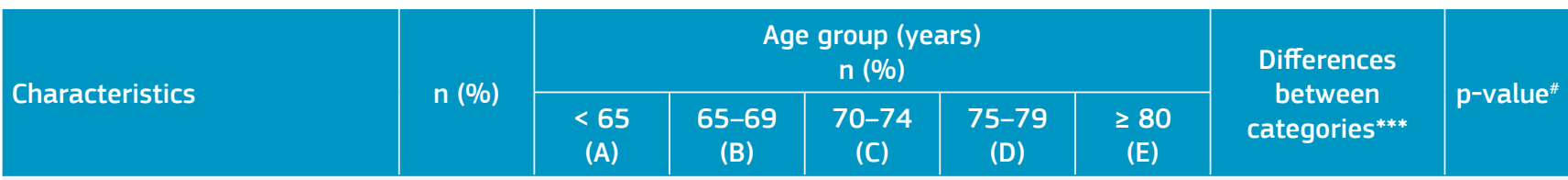

Site of the primary tumor in men $(n=309)$

\begin{tabular}{|c|c|c|c|c|c|c|c|c|}
\hline Prostate & $\begin{array}{c}177 \\
(57.28)\end{array}$ & $\begin{array}{c}23 \\
(54.76)\end{array}$ & $\begin{array}{c}36 \\
(50.00)\end{array}$ & $\begin{array}{c}47 \\
(57.32)\end{array}$ & $\begin{array}{c}33 \\
(58.93)\end{array}$ & $\begin{array}{c}38 \\
(66.67)\end{array}$ & $A=B=C=D=E$ & \multirow{3}{*}{0.066} \\
\hline Digestive system & $\begin{array}{c}69 \\
(22.33)\end{array}$ & $04(9.52)$ & $\begin{array}{c}20 \\
(27.78)\end{array}$ & $\begin{array}{c}18 \\
(22.95)\end{array}$ & $\begin{array}{c}16 \\
(28.57)\end{array}$ & $\begin{array}{c}11 \\
(19.30)\end{array}$ & $\mathrm{A}=\mathrm{B}=\mathrm{C}=\mathrm{D}=\mathrm{E}$ & \\
\hline Others* & $\begin{array}{c}63 \\
(20.39)\end{array}$ & $\begin{array}{c}15 \\
(35.72)\end{array}$ & $\begin{array}{c}16 \\
(22.22)\end{array}$ & $\begin{array}{c}17 \\
(21.73)\end{array}$ & $\begin{array}{c}07 \\
(12.50)\end{array}$ & $\begin{array}{c}08 \\
(14.03)\end{array}$ & $\mathrm{A}=\mathrm{B}=\mathrm{C}=\mathrm{D}=\mathrm{E}$ & \\
\hline
\end{tabular}

Site of the primary tumor in women $(n=299)$

\begin{tabular}{|c|c|c|c|c|c|c|c|c|}
\hline Breast & $\begin{array}{c}97 \\
(32.44)\end{array}$ & $\begin{array}{c}25 \\
(35.21)\end{array}$ & $\begin{array}{c}26 \\
(35.14)\end{array}$ & $\begin{array}{c}14 \\
(22.58)\end{array}$ & $\begin{array}{c}21 \\
(41.18)\end{array}$ & $\begin{array}{c}11 \\
(26.83)\end{array}$ & $A=B=C=D=E$ & \multirow{4}{*}{0.606} \\
\hline Digestive system & $\begin{array}{c}86 \\
(28.76)\end{array}$ & $\begin{array}{c}19 \\
(26.76)\end{array}$ & $\begin{array}{c}19 \\
(25.68)\end{array}$ & $\begin{array}{c}19 \\
(30.64)\end{array}$ & $\begin{array}{c}13 \\
(25.49)\end{array}$ & $\begin{array}{c}16 \\
(39.02)\end{array}$ & $A=B=C=D=E$ & \\
\hline Female genital system & $\begin{array}{c}73 \\
(24.42) \\
\end{array}$ & $\begin{array}{c}19 \\
(26.76)\end{array}$ & $\begin{array}{c}16 \\
(21.62) \\
\end{array}$ & $\begin{array}{c}20 \\
(32.26)\end{array}$ & $\begin{array}{c}09 \\
(17.65)\end{array}$ & $\begin{array}{c}09 \\
(22.95)\end{array}$ & $A=B=C=D=E$ & \\
\hline Others** & $\begin{array}{c}43 \\
(14.38)\end{array}$ & $\begin{array}{c}08 \\
(11.27)\end{array}$ & $\begin{array}{c}13 \\
(17.56)\end{array}$ & $\begin{array}{c}09 \\
(14.52)\end{array}$ & $\begin{array}{c}08 \\
(15.69)\end{array}$ & $\begin{array}{c}05 \\
(12.19)\end{array}$ & $A=B=C=D=E$ & \\
\hline
\end{tabular}

Metastatic disease at diagnosis

\begin{tabular}{l|c|c|c|c|c|c|c|c}
\hline Yes & $\begin{array}{c}178 \\
(29.28)\end{array}$ & $\begin{array}{c}25 \\
(22.12)\end{array}$ & $\begin{array}{c}44 \\
(30.14)\end{array}$ & $\begin{array}{c}48 \\
(33.73)\end{array}$ & $\begin{array}{c}27 \\
(25.23)\end{array}$ & $\begin{array}{c}34 \\
(34.69)\end{array}$ & $\mathrm{A}<\mathrm{C}=\mathrm{E} ; \mathrm{B}=\mathrm{D}$ & \multicolumn{1}{c|}{0.183} \\
\hline No & $\begin{array}{c}430 \\
(70.72)\end{array}$ & $\begin{array}{c}88 \\
(77.88)\end{array}$ & $\begin{array}{c}102 \\
(69.86)\end{array}$ & $\begin{array}{c}96 \\
(66.67)\end{array}$ & $\begin{array}{c}80 \\
(74.77)\end{array}$ & $\begin{array}{c}64 \\
(65.31)\end{array}$ & $\mathrm{A}>\mathrm{C}=\mathrm{E} ; \mathrm{B}=\mathrm{D}$ & \\
\hline
\end{tabular}

Functional Karnofsky Performance Scale

\begin{tabular}{|c|c|c|c|c|c|c|c|c|}
\hline$\leq 50$ & $\begin{array}{c}78 \\
(12.83)\end{array}$ & $\begin{array}{c}14 \\
(12.39)\end{array}$ & $\begin{array}{c}14 \\
(09.59)\end{array}$ & $\begin{array}{c}15 \\
(10.42)\end{array}$ & $\begin{array}{c}13 \\
(12.15)\end{array}$ & $\begin{array}{c}22 \\
(22.45)\end{array}$ & $A=B=C=D<E$ & \multirow{2}{*}{0.036} \\
\hline$>50$ & $\begin{array}{c}530 \\
(87.17)\end{array}$ & $\begin{array}{c}99 \\
(87.61)\end{array}$ & $\begin{array}{c}132 \\
(90.41)\end{array}$ & $\begin{array}{c}129 \\
(89.58)\end{array}$ & $\begin{array}{c}94 \\
(87.85)\end{array}$ & $\begin{array}{c}76 \\
(77.55)\end{array}$ & $A=B=C=D>E$ & \\
\hline \multicolumn{9}{|c|}{ Mini Nutritional Assessment } \\
\hline Normal & $\begin{array}{c}303 \\
(49.84)\end{array}$ & $\begin{array}{c}59 \\
(52.21) \\
\end{array}$ & $\begin{array}{c}80 \\
(54.79) \\
\end{array}$ & $\begin{array}{c}70 \\
(48.61)\end{array}$ & $\begin{array}{c}61 \\
(57.01) \\
\end{array}$ & $\begin{array}{c}33 \\
(33.67) \\
\end{array}$ & $A=B=C=D>E$ & \multirow{2}{*}{0.007} \\
\hline At nutritional risk & $\begin{array}{c}305 \\
(50.16)\end{array}$ & $\begin{array}{c}54 \\
(47.79)\end{array}$ & $\begin{array}{c}66 \\
(45.21)\end{array}$ & $\begin{array}{c}74 \\
(51.39)\end{array}$ & $\begin{array}{c}46 \\
(42.99)\end{array}$ & $\begin{array}{c}65 \\
(66.33)\end{array}$ & $A=B=C=D<E$ & \\
\hline \multicolumn{9}{|c|}{ Charlson Comorbidity Index } \\
\hline$<2$ comorbidities & $\begin{array}{c}455 \\
(74.84) \\
\end{array}$ & $\begin{array}{c}82 \\
(72.57) \\
\end{array}$ & $\begin{array}{c}111 \\
(76.03) \\
\end{array}$ & $\begin{array}{c}110 \\
(76.39)\end{array}$ & $\begin{array}{c}76 \\
(71.03) \\
\end{array}$ & $\begin{array}{c}76 \\
(77.55) \\
\end{array}$ & $A=B=C=D=E$ & \multirow{2}{*}{0.770} \\
\hline$\geq 2$ comorbidities & $\begin{array}{c}153 \\
(25.16)\end{array}$ & $\begin{array}{c}31 \\
(27.43)\end{array}$ & $\begin{array}{c}35 \\
(23.97)\end{array}$ & $\begin{array}{c}34 \\
(23.61)\end{array}$ & $\begin{array}{c}31 \\
(28.97)\end{array}$ & $\begin{array}{c}22 \\
(22.45)\end{array}$ & $A=B=C=D=E$ & \\
\hline
\end{tabular}

*Urinary system ( $(n=22)$, lung $(n=21)$, head and neck $(n=9)$, adrenal gland $(n=1)$, brain $(n=1)$, non-Hodgkin's lymphoma $(n=3)$, melanoma $(n=1)$, soft tissue (3), and skin $(n=2)$; **bladder $(n=7)$, brain $(n=1)$, head and neck $(n=5)$, non-Hodgkin's lymphoma $(n=1)$, melanoma $(n=3)$, soft tissue $(n=7)$, lung $(n=13)$, kidney $(n=4)$, urethra $(n=1)$, and occult primary $(n=1)$; ** Z-test; "Pearson's $\chi^{2}$ test; statistically significant values are highlighted in bold. 
KPS $(\mathrm{p}=0.036)$ and were at greater nutritional risk $(\mathrm{p}=$ $0.007)$ when compared to younger people. No differences were observed between age and tumor site, metastasis at diagnosis, and presence of comorbidities.
The evaluation of HRQoL according to age group is presented in Table 3. Among the functional scales, cognitive function showed the highest scores (mean $97.94 \pm 26.87$ ), while physical function showed the lowest ones $(68.04 \pm 28.63)$. The

Table 3. Quality of life (EORTC QLQ-C30) of older adults diagnosed with cancer, according to age group ( $N=608$ ).

\begin{tabular}{|c|c|c|c|c|c|c|c|c|}
\hline \multirow{2}{*}{ Quality of life } & \multirow{2}{*}{$\begin{array}{l}\text { Mean } \\
\text { (SD) }\end{array}$} & \multicolumn{5}{|c|}{$\begin{array}{c}\text { Age group (years) } \\
\text { Mean (SD) }\end{array}$} & \multirow{2}{*}{$\begin{array}{l}\text { Differences } \\
\text { between } \\
\text { categories** }\end{array}$} & \multirow{2}{*}{ p-value ${ }^{\#}$} \\
\hline & & $\begin{array}{c}<65 \\
(A)\end{array}$ & $\begin{array}{c}65-69 \\
\text { (B) }\end{array}$ & $\begin{array}{l}70-74 \\
\text { (C) }\end{array}$ & $\begin{array}{c}75-79 \\
\text { (D) }\end{array}$ & $\begin{array}{c}\geq 80 \\
(E)\end{array}$ & & \\
\hline Overall quality of life & $\begin{array}{l}75.34 \\
(22.77)\end{array}$ & $\begin{array}{l}72.71 \\
(25.03)\end{array}$ & $\begin{array}{c}76.02 \\
(22.12)\end{array}$ & $\begin{array}{l}74.94 \\
(21.07)\end{array}$ & $\begin{array}{c}78.89 \\
(20.68)\end{array}$ & $\begin{array}{c}74.06 \\
(25.35)\end{array}$ & $A=B=C=D=E$ & 0.322 \\
\hline \multicolumn{9}{|l|}{ Function scale* } \\
\hline Physical function & $\begin{array}{l}68.04 \\
(28.63)\end{array}$ & $\begin{array}{c}68.02 \\
(29.58)\end{array}$ & $\begin{array}{l}73.11 \\
(26.13)\end{array}$ & $\begin{array}{l}69.54 \\
(28.32)\end{array}$ & $\begin{array}{l}69.28 \\
(27.97)\end{array}$ & $\begin{array}{l}56.94 \\
(29.86)\end{array}$ & $E<A<B=C=D$ & $<0.001$ \\
\hline Global function & $\begin{array}{l}69.24 \\
(33.53)\end{array}$ & $\begin{array}{l}66.22 \\
(36.36)\end{array}$ & $\begin{array}{l}70.78 \\
(32.20)\end{array}$ & $\begin{array}{c}69.91 \\
(33.29)\end{array}$ & $\begin{array}{l}74.61 \\
(30.74)\end{array}$ & $\begin{array}{c}63.60 \\
(34.87)\end{array}$ & $A=B=C=D=E$ & 0.332 \\
\hline Cognitive function & $\begin{array}{l}87.94 \\
(26.87)\end{array}$ & $\begin{array}{l}86.43 \\
(30.09)\end{array}$ & $\begin{array}{l}87.90 \\
(26.23)\end{array}$ & $\begin{array}{c}89.81 \\
(25.02)\end{array}$ & $\begin{array}{l}88.78 \\
(24.63)\end{array}$ & $\begin{array}{l}86.05 \\
(29.08)\end{array}$ & $A=B=C=D=E$ & 0.801 \\
\hline Emotional function & $\begin{array}{l}73.52 \\
(28.41)\end{array}$ & $\begin{array}{l}64.45 \\
(33.18)\end{array}$ & $\begin{array}{c}74.31 \\
(27.27)\end{array}$ & $\begin{array}{l}75.29 \\
(27.03)\end{array}$ & $\begin{array}{l}78.82 \\
(24.61)\end{array}$ & $\begin{array}{c}74.40 \\
(28.20)\end{array}$ & $A<B=E=C<D$ & 0.003 \\
\hline Social role & $\begin{array}{l}80.53 \\
(28.17)\end{array}$ & $\begin{array}{c}80.83 \\
(28.19)\end{array}$ & $\begin{array}{c}78.31 \\
(29.58)\end{array}$ & $\begin{array}{l}81.83 \\
(28.08)\end{array}$ & $\begin{array}{l}83.49 \\
(25.65)\end{array}$ & $\begin{array}{c}78.40 \\
(28.94)\end{array}$ & $A=B=C=D=E$ & 0.565 \\
\hline \multicolumn{9}{|l|}{ Symptom scale ${ }^{* *}$} \\
\hline Fatigue & $\begin{array}{l}26.32 \\
(28.41)\end{array}$ & $\begin{array}{l}26.74 \\
(29.42)\end{array}$ & $\begin{array}{l}24.58 \\
(28.21)\end{array}$ & $\begin{array}{l}28.47 \\
(28.99)\end{array}$ & $\begin{array}{l}20.98 \\
(25.19)\end{array}$ & $\begin{array}{c}31.06 \\
(29.41)\end{array}$ & $A=B=C=D=E$ & 0.094 \\
\hline Pain & $\begin{array}{c}29.47 \\
(33.92)\end{array}$ & $\begin{array}{c}31.12 \\
(35.46)\end{array}$ & $\begin{array}{c}28.20 \\
(32.84)\end{array}$ & $\begin{array}{c}28.59 \\
(33.55)\end{array}$ & $\begin{array}{c}23.36 \\
(31.38)\end{array}$ & $\begin{array}{c}37.41 \\
(35.92)\end{array}$ & $D<A=B=C<E$ & 0.051 \\
\hline Dyspnea & $\begin{array}{c}5.70 \\
(17.23)\end{array}$ & $\begin{array}{c}5.31 \\
(16.40)\end{array}$ & $\begin{array}{c}5.03 \\
(15.82)\end{array}$ & $\begin{array}{c}4.63 \\
(17.00)\end{array}$ & $\begin{array}{c}6.23 \\
(18.39)\end{array}$ & $\begin{array}{c}8.16 \\
(19.18)\end{array}$ & $A=B=C=D=E$ & 0.569 \\
\hline Insomnia & $\begin{array}{c}28.51 \\
(37.03)\end{array}$ & $\begin{array}{c}28.32 \\
(38.37)\end{array}$ & $\begin{array}{c}33.33 \\
(38.75)\end{array}$ & $\begin{array}{c}26.39 \\
(35.01)\end{array}$ & $\begin{array}{c}23.67 \\
(33.33)\end{array}$ & $\begin{array}{c}29.93 \\
(39.32)\end{array}$ & $A=B=C=D=E$ & 0.296 \\
\hline Loss of appetite & $\begin{array}{c}25.00 \\
(36.30)\end{array}$ & $\begin{array}{c}203.3 \\
(36.97)\end{array}$ & $\begin{array}{c}21.00 \\
(35.02)\end{array}$ & $\begin{array}{c}26.39 \\
(36.95)\end{array}$ & $\begin{array}{c}19.94 \\
(30.32)\end{array}$ & $\begin{array}{c}36.39 \\
(40.35)\end{array}$ & $B=D<A=C<E$ & 0.007 \\
\hline Nausea/vomiting & $\begin{array}{c}10.94 \\
(22.16)\end{array}$ & $\begin{array}{c}11.95 \\
(23.72)\end{array}$ & $\begin{array}{c}8.45 \\
(19.69)\end{array}$ & $\begin{array}{c}13.08 \\
(24.39)\end{array}$ & $\begin{array}{c}8.87 \\
(20.26)\end{array}$ & $\begin{array}{c}12.58 \\
(22.20)\end{array}$ & $A=B=C=D=E$ & 0.300 \\
\hline Constipation & $\begin{array}{c}20.17 \\
(34.74)\end{array}$ & $\begin{array}{c}20.35 \\
(34.62)\end{array}$ & $\begin{array}{c}16.67 \\
(31.37)\end{array}$ & $\begin{array}{c}21.53 \\
(36.86)\end{array}$ & $\begin{array}{c}20.56 \\
(35.06)\end{array}$ & $\begin{array}{c}22.78 \\
(36.32)\end{array}$ & $A=B=C=D=E$ & 0.689 \\
\hline Diarrhea & $\begin{array}{c}8.17 \\
(22.90)\end{array}$ & $\begin{array}{c}8.55 \\
(24.30)\end{array}$ & $\begin{array}{c}8.90 \\
(22.24)\end{array}$ & $\begin{array}{c}6.71 \\
(21.44)\end{array}$ & $\begin{array}{c}6.71 \\
(21.83)\end{array}$ & $\begin{array}{c}10.20 \\
(25.51)\end{array}$ & $A=B=C=D=E$ & 0.757 \\
\hline Financial difficulties & $\begin{array}{c}34.21 \\
(39.06)\end{array}$ & $\begin{array}{c}35.99 \\
(39.63)\end{array}$ & $\begin{array}{c}39.27 \\
(41.92)\end{array}$ & $\begin{array}{c}29.17 \\
(36.32)\end{array}$ & $\begin{array}{c}31.15 \\
(38.38)\end{array}$ & $\begin{array}{c}35.37 \\
(38.24)\end{array}$ & $A=B=C=D=E$ & 0.213 \\
\hline
\end{tabular}

*Higher scores correspond to better quality of life; **higher scores correspond to poorer quality of life; ${ }^{* * *}$ Scheffé comparison test; ${ }^{*}$ Analysis of variance (ANOVA) test; statistically significant values are highlighted in bold; SD: standard deviation. 
highest symptom score was observed for financial difficulties (34.21 \pm 39.06$)$, followed by pain $(29.47 \pm 33.92)$, and insomnia $(28.51 \pm 37.03)$. Considering physical function, lower scores were observed in older patients aged 80 or older $(p<0.001)$. Regarding emotional function, the worst scores were obtained by younger people ( $<65$ years) $(\mathrm{p}=0.003)$. As for symptoms, older adults aged 80 or older had the lowest scores for loss of appetite $(p=0.007)$. No statistically significant differences were observed for the other function and symptom domains.

To identify potential confounders in the association between age and HRQoL, we used an univariate linear regression. Variables with $\mathrm{p}<0.20$ were selected for the adjusted model. Alcoholism was the only variable not identified as a possible confounding variable; it was not included in the testing of model fit (Table 4).

Table 5 presents the crude and adjusted analyses for the association between age and HRQoL. After adjustment, a worsening of 1.44 points was observed in the physical function $(95 \%$ confidence interval $[95 \% \mathrm{CI}]-2.73--0.16 ; \mathrm{p}=$ 0.028 ) and an improvement of 2.39 points was seen in the emotional function $(95 \% \mathrm{CI} 0.80-3.98 ; \mathrm{p}=0.003)$ with increasing age.

Table 4. Simple linear regression between potential confounding variables and HRQoL $(\mathrm{N}=608)^{*}$.

\begin{tabular}{l|c|c|c}
\multirow{2}{*}{ Variables } & Physical function & Emotional function & Loss of appetite \\
\cline { 2 - 4 } Sex & p-value & p-value & $<0.001$ \\
\hline Education & $<0.001$ & $<0.001$ & 0.628 \\
\hline Partner & 0.073 & 0.297 & 0.010 \\
\hline Family income & 0.025 & 0.002 & 0.139 \\
\hline Ethnicity (self-reported skin color) & 0.054 & 0.220 & 0.202 \\
\hline Smoking & 0.116 & 0.003 & 0.128 \\
\hline Alcoholism & 0.403 & 0.569 & 0.296 \\
\hline Tumor location & 0.757 & 0.219 & $<0.001$ \\
\hline Metastasis at diagnosis & $<0.001$ & $<0.001$ & $<0.001$ \\
\hline Charlson Comorbidity Index (CCI) & $<0.001$ & 0.001 & 0.001 \\
\hline Karnofsky Performance Status (KPS) & $<0.001$ & 0.003 & $<0.001$ \\
\hline Mini Nutritional Assessment (MNA-VR) & $<0.001$ & 0.020 & $<0.001$ \\
\hline
\end{tabular}

*Variables in bold were selected to adjust the model in the multiple linear regression.

Table 5. Crude and adjusted linear regression analysis summary of older adults diagnosed with cancer $(\mathrm{N}=608)^{*}$.

\begin{tabular}{|c|c|c|c|c|c|c|}
\hline \multirow{2}{*}{ Quality of life domains } & \multicolumn{3}{|c|}{ Crude analysis (univariate) } & \multicolumn{3}{|c|}{ Adjusted analysis (multiple) } \\
\hline & Beta & $95 \% \mathrm{Cl}$ & p-value & Beta & $95 \% \mathrm{Cl}$ & p-value \\
\hline \multicolumn{7}{|l|}{ Function scale } \\
\hline Physical function & -2.55 & $-4.24--0.85$ & 0.003 & $-1.44^{a}$ & $-2.73--0.16$ & 0.028 \\
\hline Emotional function & 2.45 & $0.77-4.13$ & 0.004 & $2.39^{b}$ & $0.80-3.98$ & 0.003 \\
\hline \multicolumn{7}{|l|}{ Symptom scale } \\
\hline Loss of appetite & 2.44 & $0.29-4.59$ & 0.026 & $1.43^{c}$ & $-0.45-3.30$ & 0.137 \\
\hline
\end{tabular}

${ }^{a}$ Adjusted for sex, Charlson Comorbidity Index, Karnofsky Performance Status, and Mini Nutritional Assessment; 'badjusted for sex, Mini Nutritional Assessment, metastasis at diagnosis, and ethnicity; 'adjusted for sex, Mini Nutritional Assessment, and metastasis at diagnosis; *statistically significant values are highlighted in bold; 95\%Cl: confidence interval of 95\%. 


\section{DISCUSSION}

This study examined HRQoL according to age group in 608 older patients with cancer treated at a single referral center in northeast Brazil.

Considering the demographic characteristics of this population, most patients had low education levels, a family income of less than twice the minimum wage (in 2015/2016 the minimum wage in Brazil was $U \$ 250 / 270$ per month), and non-white skin color (self-reported), which is in agreement with the characteristics of the Brazilian older adult population seeking cancer treatment in the public health system. ${ }^{18,19}$

The most frequent cancer topographies in this population were male genital organs, digestive organs, and female breasts, which reflected the estimated incidence of new cancer cases in the male and female populations during the study period. ${ }^{20}$

We also observed that almost one-third of the patients had metastases at diagnosis. In Brazil, the delay in cancer diagnosis has been attributed to a poor access to health services and to the lack of public health policies urging the early diagnosis of this disease. ${ }^{21-23}$

Among subjects aged $<65$ years, most of the cases were women. This may be partly explained by the topographical distribution of cancer in the country. ${ }^{20} \mathrm{~A}$ high frequency of breast cancer in younger women and of prostate cancer in older men has also been described by other authors. ${ }^{24}$ Moreover, we observed that older adults more often had lower education levels and were not living with a partner. In contrast, Akechi et al. ${ }^{25}$ found no differences in education and marital status between older adults and younger older adults. This discrepancy may be related to the sociodemographic profile of the Brazilian older adult population. ${ }^{18}$

Regarding clinical characteristics, patients in this study who were aged 80 or older showed greater impairment in KPS ( $p=0.036)$ and were at higher nutritional risk measured by the MNA-SF ( $p=0.007$ ) compared to younger older adults. Aging involves biological phenomena associated with the accumulation of molecular and cellular damage, which in time lead to a gradual loss of physiological and immunological reserves and of the individual's intrinsic capabilities. ${ }^{25}$ Moreover, aging often implicates, in addition to biological losses, changes in social roles and positions linked to psychological and cultural aspects that interact with the reality in which the individual lives and reflect on their overall health. ${ }^{1,26}$ In this context, performance status and nutritional risk are expected to be compromised in aging populations.

In the present study, "older older" participants had worse HRQoL in the physical function domain $(p=0.028)$ in comparison to "younger older" patients. Similar results have been reported in various social, cultural, and economic realities. In
Germany, a study comparing older adults by age group using the same questionnaire (EORTC QLQ-C30) also found greater impairment of the physical function domain in older adults. ${ }^{27}$ In addition, a meta-analysis of randomized controlled clinical trials performed in Europe ${ }^{14}$ and a randomized study conducted in Japan ${ }^{25}$ reported worse physical function with advancing age.

Our results also indicated a worse emotional function score among younger participants $(<65$ years $)(\mathrm{p}=0.003)$. Similarly, Akechi et al. ${ }^{25}$ also showed worse HRQoL scores regarding emotional function in patients under 70 years of age $(\mathrm{p}<0.01)$. On the other hand, Baumann et al. ${ }^{27}$ revealed a similarity in emotional function between patients aged 60 to 69 years and those aged $\geq 70$ years. The same was observed in an analysis by Quinten et al., ${ }^{13}$ where there was no association between age and emotional function. Other studies also observed decreased cognitive function ${ }^{27}$ and constipation ${ }^{13}$ in older adults. Social function was also better in those aged 70 years or older in a study by Akechi et al. ${ }^{25}$ Different authors have suggested that changes in psychological symptoms have less impact on the daily activities of older adults when compared to younger older adults. ${ }^{28}$

In this study, greater loss of appetite was observed in those aged 80 years or more only in the univariate analysis. Baumann et al. ${ }^{27}$ and Quinten et al. ${ }^{13}$ also reported greater loss of appetite in patients aged 70 years or more. In a study conducted in 31 centers in the United Kingdom involving 2208 women with early-stage breast cancer, age had no influence on loss of appetite. ${ }^{29}$ Baumann et al. ${ }^{27}$ showed that older age was associated with a worse quality of life regarding the following symptoms: fatigue, nausea and vomiting, pain, dyspnea, and constipation. In contrast, Akechi et al. ${ }^{25}$ found no difference in HRQoL between age groups. However, their study showed a lower impact of nausea and vomiting and of financial difficulties in patients aged 70 years or more.

This study's main strength is the fact that age was stratified in several groups (considering the different older adult settings available), which contributes to a better understanding of the influence of age on HRQoL in cancer patients. Our results showed an association between metastatic disease at admission, KPS, CCI, and MNA in HRQoL. Therefore, these variables were used in the final model as adjustment variables in the association between HRQoL and the age groups. In this scenario, we were able to identify, at the time of cancer diagnosis, vulnerable older adult groups who required special attention in clinical practice. The results of this research reflect the importance of studying older adults with cancer considering their sociodemographic and clinical characteristics and based on HRQoL in order to favor the assistance, care, and impact of treatments on the survival of older adults. 
On the other hand, limitations of this study are related to the heterogeneity of the sample (with a large variability in tumor sites), which could have compromised the analysis and interpretation of HRQoL functions and symptoms. In contrast, this limitation can be regarded as a strength since it reflects the reality of the care of patients with cancer in Brazil. In addition, the cross-sectional approach with data collection at a time close to the diagnosis prevented the knowledge of the impact of treatments on HRQoL and limited the analysis of HRQoL for each therapeutic approach. Another possible limitation was the use of instruments that may not describe functional state in older patients (KPS) and the use of the CCI. However, the choice of these instruments considered the possibility of comparing our results with international studies, since these are widely used tools, as well as the previous experience of the team where data were collected. Considering the results of this research, the promotion of strategies directed to the physical function of older people could help improve the HRQoL of this population.

\section{CONCLUSION}

In conclusion, older adults aged 80 years or older showed poor performance status and were at higher nutritional risk when compared to younger older adults. Greater impact on HRQoL was observed only in physical function, while the lowest impairment was described in cognitive function. In the symptom scale, financial difficulties exerted the most influence on HRQoL, followed by pain and sleep disorders. After adjustment, a worsening was observed in physical function and an improvement was detected in emotional function with increasing age.

\section{CONFLICTS OF INTEREST}

The authors declare no conflicts of interest.

\section{FUNDING}

This research did not receive any specific grant from funding agencies in the public, commercial, or not-for-profit sectors.

\section{AUTHORS' CONTRIBUTION}

CRCX: conceptualization, data curation, investigation, writing - original draft. AB: conceptualization, formal analysis, methodology, supervision, writing — review \& editing. JTOL: data curation, investigation, validation, visualization, writing - review \& editing. ALC: data curation, investigation, validation, visualization, writing - review \& editing. MCB: conceptualization, formal analysis, methodology, supervision, writing — review \& editing. MJGM: conceptualization, formal analysis, methodology, supervision, writing — review \& editing. LCST: conceptualization, formal analysis, methodology, supervision, writing — review \& editing.

\section{REFERENCES}

1. Mathers CD, Stevens GA, Boerma T, White RA, Tobias MI. Causes of international increases in older age life expectancy. Lancet. 2015;385(9967):540-8. https://doi.org/10.1016/s01406736(14)60569-9

2. World Health Organization. World report on ageing and health. Geneva: World Health Organization; 2015. Available at: http://apps. who.int/iris/bitstream/10665/186463/1/9789240694811_eng.pdf. Accessed on: Apr 20, 2016

3. Giantin V, Falci C, De Luca E, Valentini E, lasevoli M, Siviero P, et al. Performance of the Multidimensional Geriatric Assessment and Multidimensional Prognostic Index in predicting negative outcomes in older adults with cancer. Eur J Cancer Care (Engl). 2018;27(1). https://doi.org/10.1111/ecc.12585

4. Instituto Brasileiro de Geografia e Estatística. Síntese de indicadores sociais: uma análise das condições de vida da população brasileira. Instituto Brasileiro de Geografia e Estatística; 2013. Available at: https://biblioteca.ibge.gov.br/visualizacao/livros/liv66777.pdf. Accessed on: Feb 10, 2017.

5. Fitzpatrick TR, Edgar L, Holcroft C. Assessing the relationship between physical fitness activities, cognitive health, and quality of life among older cancer survivors. J Psychosoc Oncol. 2012;30(5):556-72. https:// doi.org/10.1080/07347332.2012.703768

6. Blakely AM, Chanson D, Wong FL, Eng OS, Sentovich SM, Melstrom KA, et al. Health-related quality of life and oncologic outcomes after surgery in older adults with colorectal cancer. Support Care Cancer. 2019;28:2857-65. https://doi.org/10.1007/s00520-019-05087-3
7. World Health Organization. WHOQOL - Measuring quality of life Geenva: World Health Organization; 1997. Available at: http://www. who.int/mental_health/media/68.pdf. Accessed on: Apr 2, 2015.

8. O’Donovan A, Mohile SG, Leech M. Expert consensus panel guidelines on geriatric assessment in oncology. Eur J Cancer Care (Engl). 2015;24(4):574-89. https://doi.org/10.1111/ecc.12302

9. Yucel B, Akkas EA, Okur Y, Eren AA, Eren MF, Karapinar $\mathrm{H}$, et al. The impact of radiotherapy on quality of life for cancer patients: a longitudinal study. Support Care Cancer. 2014;22(9):2479-87. https://doi.org/10.1007/s00520-014-2235-y

10. Adam S, Doege D, Koch-Gallenkamp L, Thong MSY, Bertram H, Eberle A, et al. Age-specific health-related quality of life in diseasefree long-term prostate cancer survivors versus male population controls-results from a population-based study. Support Care Cancer. 2019;28:2875-85. https://doi.org/10.1007/s00520-019-05120-5

11. Fiteni F, Vernerey D, Bonnetain F, Vaylet F, Sennélart H, Trédaniel J, et al. Prognostic value of health-related quality of life for overall survival in elderly non-small-cell lung cancer patients. Eur J Cancer. 2016:52:120-8. https://doi.org/10.1016/j.ejca.2015.10.004

12. Wildiers H, Mauer M, Pallis A, Hurria A, Mohile SG, Luciani A, et al. End points and trial design in geriatric oncology research: $A$ joint European Organization for Research and treatment of cancer Aliance for Clinical Trials in Oncology- International Society of Geriatric Oncology Position Article. J Clin Oncol. 2013;31(29):3711-8. https://doi.org/10.1200/jco.2013.49.6125 
13. Quinten C, Coens C, Guislain I, Zikos E, Sprangers MAG, Ringash J, et al. EORTC Clinical Groups. The effects of age on healthrelated quality of life in cancer populations: A pooled analysis of randomized controlled trials using the European Organisation for Research and Treatment of Cancer (EORTC) QLQ-C30 involving 6024 cancer patients. Eur J Cancer. 22015;51(18):2808-19. https:// doi.org/10.1016/j.ejca.2015.08.027

14. Quinten C, Coens C, Mauer M, Comte S, Sprangers MAG, Cleeland $C$, et al. Baseline quality of life as a prognostic indicator of survival: A meta-analysis of individual patient data from EORTC clinical trials. Lancet Oncol. 2009;10(9):865-71. https://doi.org/10.1016/ s1470-2045(09)70200-1

15. Sanoff HK, Goldberg RM, Pignone MP. A systematic review of the use of quality of life measures in colorectal cancer research with attention to outcomes in elderly patients. Clin Colorectal Cancer. 2007;6(10):700-9. https://doi.org/10.3816/CCC.2007.n.039

16. Aaronson NK, Ahmedzai S, Bergman B, Bullinger M, Cull A, Duez NJ, et al. The European Organization for Research and treatment of cancer QLQ-C30: a quality of life instrument for use in international clinical trials in oncology. J Natl Cancer Inst. 1993;85(5):365-76. https://doi.org/10.1093/jnci/85.5.365

17. Franceschini J, Jardim JR, Fernandes ALG, Jamnik S, Santoro IL. Reprodutibilidade da versão em português do Brasil do European Organization for Research and Treatment of Cancer Core Quality of Life Questionnaire em conjunto com seu módulo específico para câncer de pulmão. J Bras Pneumol. 2010;36(5):595-602. https://doi. org/10.1590/S1806-37132010000500011

18. Pacagnella ABB, Santana EDB, Gome MM, Fattori A, Guariento ME. Sociodemographic profile of elderly patients with cancer in a tertiary care service of SUS. Geriatr Gerontol Aging. 2014;8(1):27-31.

19. Boccolini CS, Souza-Junior PRB. Inequities in health care utilization: Results of the Brazilian National health survey. Int J Equity Health. 2016;15(1):150. https://doi.org/10.1186/s12939-016-0444-3

20. Instituto Nacional do Câncer José de Alencar Gomes da Silva. Estimativa 2016: incidência de câncer no Brasil. Rio de Janeiro: Instituto Nacional do Câncer José de Alencar Gomes da Silva; 2015. Available at: https://www.inca.gov.br/bvscontrolecancer/publicacoes/ edicao/Estimativa_2016.pdf. Accessed on: Jun 10, 2016.
21. Thuler LCS, Aguiar SS, Bergmann A. Determinants of late stage diagnosis of cervical cancer in Brazil. Rev Bras Ginecol Obstet. 2014;36(6):237-43. https://doi.org/10.1590/S0100720320140005010

22. Abrahão KS, Bergmann A, Aguiar SS, Thuler LC. Determinants of advanced stage presentation of breast cancer in 87,969 Brazilian women. Maturitas. 2015;82(4):365-70. https://doi.org/10.1016/j. maturitas.2015.07.021

23. Costa G, Thuler LCS, Ferreira CG. Epidemiological changes in the histological subtypes of 35,018 non-small-cell lung cancer cases in Brazil. Lung Cancer. 2016;97:66-72. https://doi.org/10.1016/j. lungcan.2016.04.019

24. Gundrum JD, Go RS. Cancer in the oldest old in the United Sates: Current statistics and projections. J Geriatric Oncol. 2012;3(4):299306. https://doi.org/10.1016/j.jgo.2012.08.003

25. Akechi T, Okuyama T, Uchida M, Nakaguchi T, Ito Y, Yamashita H, et al. Perceived Needs, Psychological Distress and Quality of Life of Elderly Cancer patients. Jpn J Clin Oncol. 2012;42(8):704-10. https://doi.org/10.1093/jjco/hys075

26. Falandry C, Bonnefoy M, Freyer G, Gilson E. Biology of cancer and aging: a complex association with cellular senescence. J Clin Oncol. 2014;32(24):2604-10. https://doi.org/10.1200/jco.2014.55.1432

27. Baumann R, Pütz C, Röhrig B, Höffken K, Wedding U. Health-related quality of life in elderly cancer patients, elderly non-cancer patients and elderly general population. Eur J Cancer Care. 2009;18(5):45765. https://doi.org/10.1111/j.1365-2354.2008.00975.x

28. Mitchell AJ, Chan M, Bhatti H, Halton M, Grassi L, Johansen C, et al. Prevalence of depression, anxiety and adjustment disorder in oncological, haematological, and palliative-care setting: a meta-analysis of 94 interview-based studies. Lancet Oncol. 2011;12(12):160-74. https://doi.org/10.1016/s14702045(11)70002-x

29. Hopwood P, Haviland J, Mills J, Sumo G, Bliss JM. Trial Management Group. The impact of age and clinical factors on quality of life in early breast cancer: an analysis of 2208 women recruited to the UK start Trial (Standardisation of Breast Radiotherapy Trial). Breast. 2007;16(3):241-51. https://doi.org/10.1016/j.breast.2006.11.003 